\title{
La temperatura de fase en la transición de nieve a lluvia: definición y aplicación al cálculo de la cota de nieve
}

https://doi.org/10.31978/639-19-010-0.121

\author{
Alberto Fernández Matía ${ }^{1}$ (afernandezm@aemet.es) \\ Álvaro Subías Díaz-Blanco ${ }^{1}$ (asubiasd@aemet.es)
}

'AEMET / Dirección de Producción e Infraestructuras / Área de Técnicas y Aplicaciones de Predicción

\begin{abstract}
RESUMEN
Se analiza la transición de nieve a lluvia mediante nuevas adaptaciones de la ecuación de fusión de los copos y de la ley de distribución de sus tamaños en una nevada.

El análisis introduce dos nuevas definiciones, la de temperatura de fase, que representa el forzamiento atmosférico instantáneo, y la de área de fase, que, al calcular el agua fundida en el copo, integra este forzamiento. Al tener en cuenta la distribución de tamaños de los copos, se construye finalmente una última variable, a la que llamamos fase $\phi$, con la que se calcula la proporción de los que, a una altitud dada, no han llegado a fundirse del todo.

La naturaleza binaria de la transición de lluvia a nieve permite aplicar la regresión logística a $\phi$ para obtener la probabilidad de que, a una altitud dada, una nevada no se haya transformado todavía en lluvia. El cálculo de la cota de nieve surge de forma natural a partir de esta probabilidad.
\end{abstract}

PALABRAS CLAVE: nieve; lluvia; cambio de fase; tipos de precipitación; banda brillante; cota de nieve.

\section{INTRODUCCIÓN}

El hecho de que las precipitaciones sean en forma de nieve, o de lluvia, condiciona de forma muy distinta tanto la actividad humana como la ecología de un lugar (HARPOLD et al., 2017).

En este artículo se propone una forma nueva de calcular la probabilidad de que, a una altitud dada, una nevada no se haya transformado aún en lluvia (probabilidad de nevada en adelante). El cálculo se hace a partir de una nueva variable, la fase $\phi$, que permite obtener la proporción de copos que no han llegado a fundirse del todo (proporción de copos en adelante). La cota de nieve, definida como la altitud límite de la transición de nieve a lluvia, surge de forma natural a partir de la probabilidad de nevada.

Aunque únicamente teórico, ya que la propuesta de cálculo de la probabilidad de nevada no está verificada todavía con datos de observación, el artículo presenta definiciones y desarrollos originales. Esta teoría se ha implementado en los modelos de predicción numérica Harmonie-Arome y HRES-IFS del ECMWF, y se aplica ya, tanto al cálculo de la cota de nieve como al de la probabilidad de nevada, en los productos de sondeos de AEMET. 


\section{CONTENIDO Y DESARROLLO TEÓRICO}

Se citan a continuación los apartados teóricos del artículo con una breve descripción de su contenido:

3. Ecuación de fusión del copo de nieve. Temperatura de fase. A partir de esta ecuación se introduce el nuevo concepto de temperatura de fase, que representa el forzamiento atmosférico instantáneo. La cota de inicio de la fusión se corresponde con la isocero de esta temperatura de fase.

4. Transición de fase en un copo. Área de fase. La integración, a partir de la cota de inicio, de la ecuación de fusión permite seguir la evolución de la proporción de agua líquida fundida en el copo (proporción líquida en adelante) a lo largo de su descenso. La integral de la dependencia atmosférica del agua líquida fundida se recoge en otra nueva definición: el área de fase.

5. Transición de nieve a lluvia. Proporción de copos. La proporción de copos en una nevada se puede calcular conociendo el diámetro del mayor copo fundido y la distribución de diámetros. Llamamos fase $\phi$ a la variable que obtenemos para calcular esta proporción.

6. Propuesta de cálculo de la probabilidad de nevada y de la cota de nieve. Se propone un método de cálculo de la probabilidad de nevada basado en la ecuación logística aplicada a $\phi$. La cota de nieve se redefine como la altitud a la que la probabilidad de nevada es del cincuenta por ciento.

En el apartado 7 se citan las Aplicaciones que se han desarrollado al implementar la teoría, y en el 8 se formulan las Conclusiones.

\section{ECUACIÓN DE FUSIÓN DEL COPO DE NIEVE. TEMPERATURA DE FASE}

Un copo de nieve empieza a fundir cuando su temperatura alcanza la de la fusión del hielo $\left(T_{0}=0{ }^{\circ} \mathrm{C}\right)$, y se mantiene a esta temperatura constante $T_{0}$ mientras duren los cambios de fase (fusión o recongelación). Si $T$ es la temperatura del aire, el forzamiento térmico que experimenta el copo durante la transición de fase es proporcional a $T-T_{0}$, y el debido a la humedad proporcional a $\rho_{v}-\rho_{v, \text { sat }}\left(T_{0}\right)$ ( $\rho_{v}$ es la densidad del vapor de agua del aire y $\rho_{v, \text { sat }}\left(T_{0}\right)$ la correspondiente saturada a $\left.T_{0}\right)$. La variación del agua líquida fundida $m_{w}$ respecto al tiempo $\tau$, se puede expresar como (Mitra et al., 1990):

$$
L_{m} \frac{d m_{w}}{d \tau}=4 \pi \bar{f} C_{i}\left[K_{a}(T)\left(T-T_{0}\right)+L_{e} D_{v}(T, p)\left(\rho_{v}-\rho_{v, s a t}\left(T_{0}\right)\right)\right]
$$

$L_{m}$ es el calor latente de fusión del hielo, $\bar{f}$ el coeficiente de ventilación del copo (se suponen iguales los coeficientes de ventilación de calor y de evaporación), $C_{i}$ su capacitancia, $K_{a}$ la conductividad térmica del aire, $L_{e}$ el calor latente de evaporación del agua a $T_{0}, D_{v}$ la difusividad del vapor de agua en el aire y $p$ la presión atmosférica.

Si dividimos la dependencia atmosférica (la expresión entre corchetes de esta ecuación) por la constante $K_{a}\left(T_{0}\right)$, obtenemos una expresión con dimensión de temperatura que denominamos temperatura de fase $t_{\phi}$ :

$$
t_{\phi}=\frac{1}{K_{a}\left(T_{0}\right)}\left[K_{a}(T)\left(T-T_{0}\right)+L_{e} D_{v}(T, p)\left(\rho_{v}-\rho_{v, s a t}\left(T_{0}\right)\right)\right]
$$

$t_{\phi}$ representa, por lo tanto, la dependencia atmosférica instantánea del cambio de fase del copo: vemos (figura 1) que funde ( $m_{w}$ aumenta) si $t_{\phi}$ es positiva, y hay recongelación del agua fundida ( $m_{w}$ disminuye) cuando $t_{\phi}$ es negativa. La altitud $z_{0}$ que nos marca el inicio de la fusión es la isocero de $t_{\phi}, t_{\phi}\left(z_{0}\right)=0$, por lo que solo $z_{0}$ depende del perfil atmosférico (no de las características del copo) y se concluye que en una nevada todos los copos inician su fusión a la misma altitud $z_{0}$. 


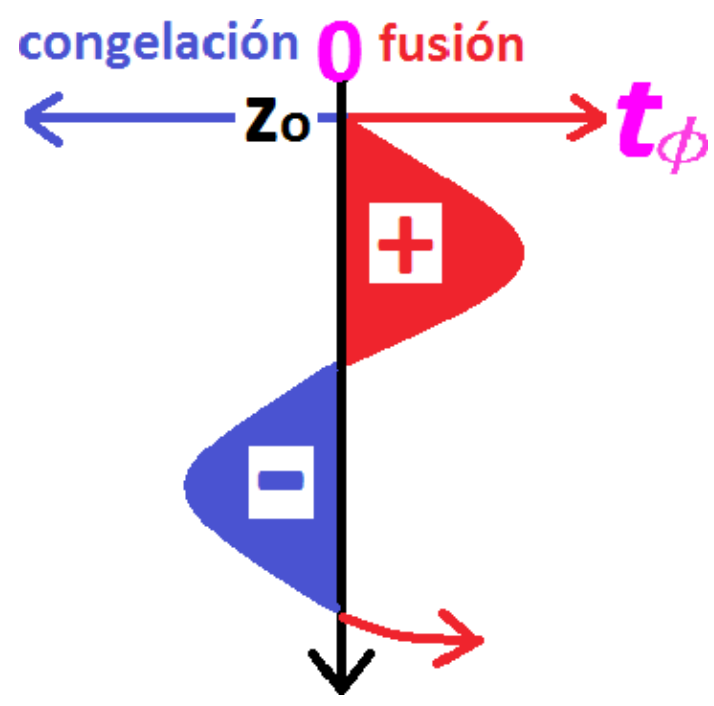

Figura 1. Cota de inicio $z_{0}$ de la fusión del copo y zonas de fusión y recongelación en función de la temperatura de fase $t_{\phi}$.
Si $t=\left(T-T_{0}\right)$ es la temperatura del aire en ${ }^{\circ} \mathrm{C}$, podemos expresar la conductividad atmosférica como (ANDREAS, 2005):

$K_{a}(T)=K_{a}\left(T_{0}\right) k(t)$

$K_{a}\left(T_{0}\right)=2,411 \cdot 10^{-2} \mathrm{~W} \mathrm{~m}^{-1}{ }^{\circ} \mathrm{C}^{-1}$

$k(t)=\left(1+3,309 \cdot 10^{-3} t-1,441 \cdot 10^{-4} t^{2}\right)$

y la difusividad del vapor de agua en el aire $D_{v}(T, p)$ como (Hall y PruPPacher, 1976):

$D_{v}(T, p)=D_{v}\left(T_{0}, p_{0}\right)\left(T / T_{0}\right)^{1,94}\left(p_{0} / p\right)$

$D_{v}\left(T_{0}, p_{0}\right)=2,11 \cdot 10^{-5} \mathrm{~m}^{-2} \mathrm{~s}^{-1}$

$p_{0}=1013,25 \mathrm{hPa}$

Trasladando las expresiones anteriores y la de $\rho_{v}$ en función de la humedad relativa $h, \rho_{v}=h \rho_{v, \text { sat }}\left(T_{0}\right)$, podemos reescribir $t_{\phi}$ :

$$
\begin{gathered}
t_{\phi}(t, h, p)=k(t) t+t_{c}\left(1+t / T_{0}\right)^{1,94}\left(p_{0} / p\right)\left(h \rho_{v, \text { sat }}(t) / \rho_{v, \text { sat }}\left(T_{0}\right)-1\right) \\
t_{c}=L_{e} D_{v}\left(T_{0}, p_{0}\right) \rho_{v, \text { sat }}\left(T_{0}\right) / K_{a}\left(T_{0}\right)
\end{gathered}
$$

donde $t_{c}$ es una constante de valor aproximado $10,6^{\circ} \mathrm{C}$.

La isocero $t_{\mathrm{\phi}}(t, h, p=$ cte. $)=0$ determina, para una presión constante dada, la curva $h=h(t)$ que relaciona la humedad y la temperatura en el momento de la fusión o recongelación, y divide el espacio $(t, h)$ entre las áreas de fusión y de recongelación. La figura 2 dibuja $h=h(t) \operatorname{con} p=p_{0}$.

\section{Notas sobre la temperatura de fase}

- La isocero de la temperatura de fase $\left(t_{\phi}(t, h, p)=0\right)$, que determina la cota de inicio de la fusión, coincide, a efectos prácticos, con la isocero del termómetro de hielo (este se define como el termómetro húmedo pero cambiando el calor latente de evaporación del agua por el de sublimación). De lo anterior se deduce que la cota de inicio de la fusión no difiere mucho de la isocero del termómetro húmedo (menos cuanto más alta es la humedad).

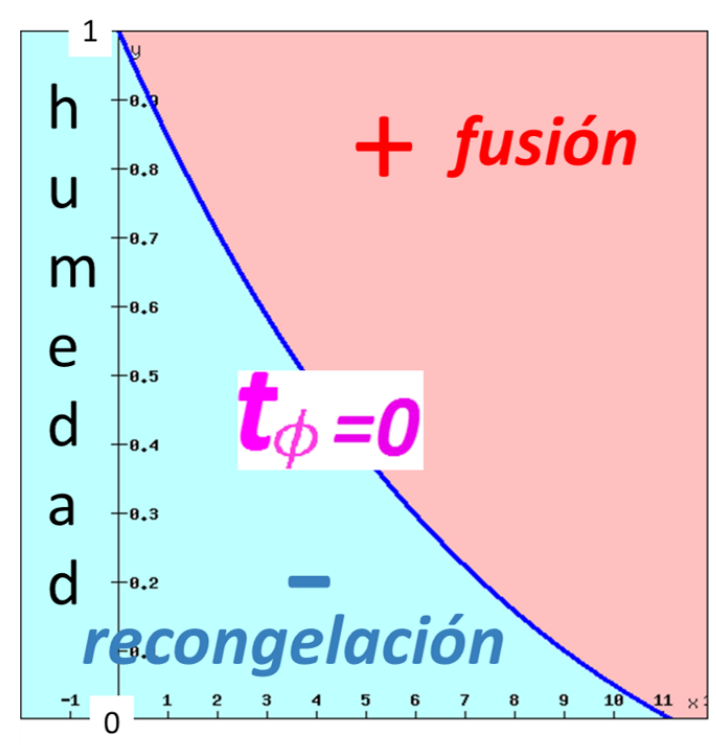

te mperatura
- Como se puede deducir a partir de sus definiciones, se observa en los sondeos que las líneas de la temperatura de fase y de la temperatura equivalente son aproximadamente paralelas.

- Si consideramos que la humedad es constante, se puede ver que, a efectos prácticos, el gradiente de la temperatura de fase con la altitud también lo es, con un valor aproximado de $-11^{\circ} \mathrm{C} \mathrm{km}^{-1}$.

Figura 2.

Curva $h=h(t)$, de humedad en función de la temperatura, para la isocero de la temperatura de fase $t_{\phi}$ y presión constante $p_{0}=1013,25 \mathrm{hPa}$. La curva divide el plano entre la zona de fusión ( $t_{\phi}$ positiva) y de recongelación ( $t_{\phi}$ negativa). 


\section{TRANSICIÓN DE FASE EN UN COPO. ÁREA DE FASE}

Vamos a obtener la ecuación [1] que nos da, para una altitud dada $z$ de la capa de fusión del copo (desde la cota de inicio de la fusión con $l\left(z_{0}\right)=0$, hasta su fusión total en $z_{1}, l\left(z_{1}\right)=1$ ), la proporción líquida $l=l(z)$ (figura 3 ). Suponemos, por simplicidad (véase la nota al final), que el copo no se recongela durante el proceso.

Sea $M$ la masa del copo, $l=m_{w} / M$ su proporción líquida, y $D$ su diámetro equivalente (definido mediante la igualdad $M=\pi \rho_{\omega} D^{3} / 6$, donde $\rho_{\omega}$ es la densidad del agua). Si expresamos la velocidad de caída del copo, $v=d z / d \tau$, como (Heymsfield et al., 2006):

$$
v=v_{F} /\left[\left(p T_{0}\right) /\left(p_{0} T\right)\right]^{b}
$$

(donde $\left[\left(p T_{0}\right) /\left(p_{0} T\right)\right]$ es la densidad relativa del aire y $\underline{b}=0,54)$, y descomponemos (SZYRMER y ZAWADZKi, 1999) $f C_{i} / v_{F}=f(l) D^{F}(\operatorname{con} F=1,1)$, entonces [1] se puede reescribir así:

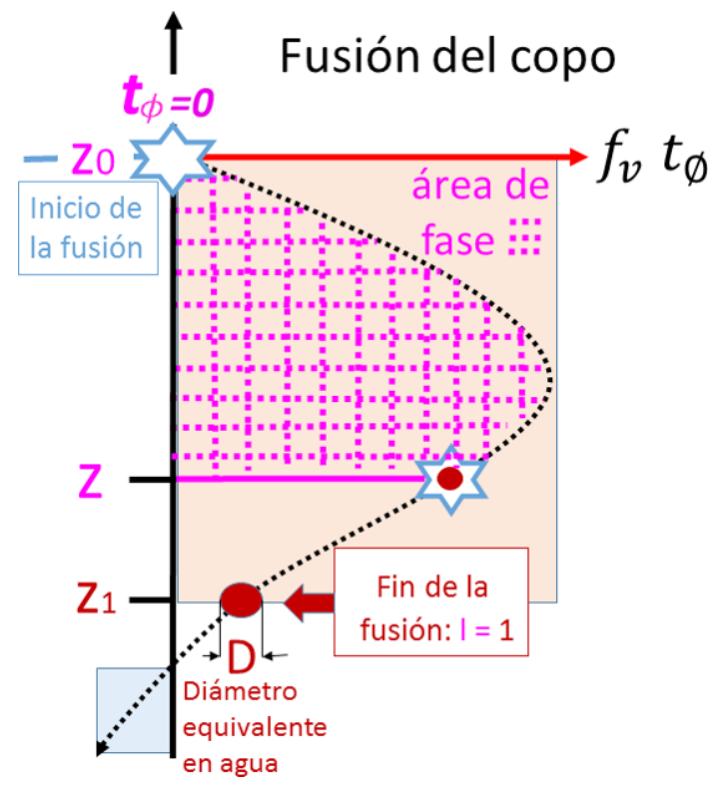

Figura 3. Área de fase (cuadrícula en magenta) en la evolución del copo en la capa de la fusión.

$$
\left(\frac{p}{p_{0}} \frac{T_{0}}{T}\right)^{b} t_{\phi}(t, h, p) d z=D^{3-F} \frac{L_{m} \rho_{\omega}}{24 K_{a}\left(T_{0}\right)} \frac{d l}{f(l)}
$$

Integrando [7] desde la cota de inicio de la fusión $z_{0}\left(l\left(z_{0}\right)=0\right)$ hasta la altitud $z(l=l(z))$, y definiendo

$$
\begin{gathered}
A_{\phi}(z)=\int_{z}^{z_{0}}\left(\frac{p}{p_{0}} \frac{T_{0}}{T}\right)^{b} t_{\phi}(t, h, p) d z \\
L(l)=\frac{L_{m} \rho_{\omega}}{24 K_{a}\left(T_{0}\right)} \int_{0}^{l} \frac{d l}{f(l)}
\end{gathered}
$$

se obtiene finalmente la ecuación buscada:

$$
A_{\phi}(z)=D^{3-F} L(l)
$$

A $A_{\phi}(z)$, que depende solo del perfil atmosférico, la denominamos área de fase, y puede ser interpretada como el calor que recibe el copo mientras funde. $L(l)$ es una función creciente de la proporción líquida $l=l(z)$. La expresión de $L(l)$ es distinta para cada tipo de copo.

Conociendo $z_{0}$ y haciendo $l=1$ en [10], se puede obtener la altitud $z_{1}$ a la que funde un copo de diámetro $D$, convirtiéndose en gota de lluvia:

$$
A_{\phi}\left(z_{1}\right)=L(1) D^{3-F}
$$

y, en una nevada, el diámetro $D_{1}(z)$ de la mayor gota (copo completamente fundido) que podemos encontrar a la altitud $z$ :

$$
\begin{gathered}
D_{1}(z)=\left(A_{\phi}(z) / L(1)\right)^{\delta} \\
\delta=1 /(3-F)
\end{gathered}
$$

Nota. La ecuación [10] también es aplicable en intervalos de recongelación o nuevos intervalos posteriores de fusión, siempre que conozcamos el valor del parámetro y la expresión de la función válidos en cada intervalo. 


\section{TRANSICIÓN DE NIEVE A LLUVIA. PROPORCIÓN DE COPOS}

La altitud a la que funde un copo se puede calcular conociendo su diámetro con la ecuación [11]. En una nevada los copos, aun siendo del mismo tipo, tienen distintos diámetros, por lo que, para seguir analizando la transición de nieve a lluvia, hay que tener en cuenta su distribución.

Si consideramos (SEKHON y SRIVASTAVA, 1969) una distribución exponencial de sus diámetros con parametrización de Marshall-Palmer, y que la mayor gota (copo completamente fundido) a la altitud tiene un diámetro equivalente $D_{1}$, podemos expresar la proporción de copos como

$$
p_{F}(z)=\exp \left(-\lambda D_{1}(z) / R^{\gamma}\right)
$$

donde $R$ es la intensidad de la precipitación en $\mathrm{mm} / \mathrm{h}$, $\lambda=22,9 \mathrm{~cm}^{-1}$ y $\gamma=0,45$ para la nieve no granulada.

Sustituyendo [12a] en [13] obtenemos

$$
p_{F}(z)=\exp \left(-\frac{\lambda}{(L(1))^{\delta}} \frac{\left(A_{\phi}(z)\right)^{\delta}}{R^{\gamma}}\right)
$$

Vemos que la proporción de copos depende de la intensidad de precipitación, de tal forma que cuanto más copiosa es una nevada más tarda en convertirse en lluvia (véase la figura 4).

Llamamos fase $\phi$ al cociente

$$
\left.\phi(z)=\left(A_{\phi}(z)\right)^{\delta} / R^{\gamma}\right)
$$

que recoge la dependencia meteorológica de $p_{F}$. $\phi$ se estandariza para los cálculos expresando $A_{\phi}$ en ${ }^{\circ} \mathrm{C}$ m y $R$ en mm/h.

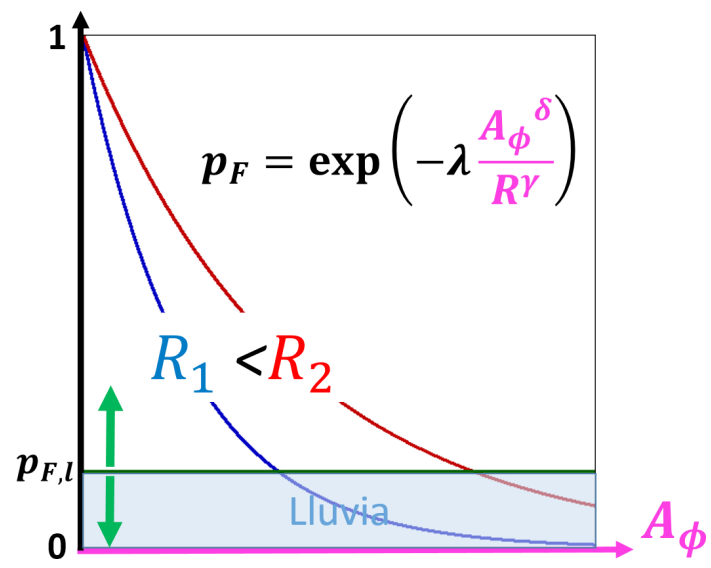

Figura 4. Proporción de copos $p_{F}$ en función del área de fase $A_{\phi}$ para dos valores distintos, $R_{1}<R_{2}$, de la intensidad de precipitación.

La proporción $p_{F, l}$ separa las zonas de nevada y lluvia.

\section{PROPUESTA DE CÁlCULO DE LA PROBABILIDAD DE NEVADA Y DE LA COTA DE NIEVE}

La cota de nieve se define (Del Hoyo et al., 2012) como la altitud límite de la nevada, es decir, de la transición de nieve a lluvia. Esta definición lleva implícita una incertidumbre en la fijación del límite, que cada observador puede resolver de forma distinta (figura 5). Una forma de superar esta incertidumbre es la de fijar una proporción de copos $p_{F, l}$ (figura 4) por debajo de la cual decimos que llueve, pero el problema está en elegir esa proporción.

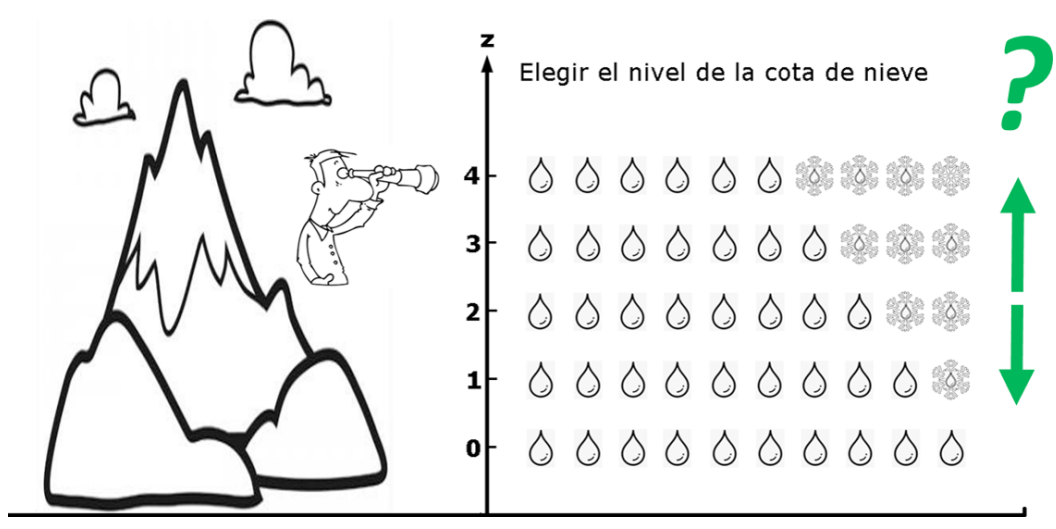

Figura 5. Incertidumbre en la observación de la cota de nieve. 
La incertidumbre se puede resolver, o bien en términos estadísticos, definiendo la cota como la mediana de un conjunto de observaciones (la mitad la sitúan por encima y la otra mitad por debajo), o bien dando la definición equivalente en términos de probabilidad: la probabilidad de que observemos nevada por encima de la cota es del $50 \%$.

Dada la naturaleza binaria de la transición de nieve a lluvia podemos abordar el cálculo de la probabilidad de nevada $p_{s}$ mediante regresión logística. La fase $\phi$, que recoge la dependencia meteorológica de la proporción de copos, es un buen candidato como predictor de la regresión. Por todo ello, se propone la siguiente fórmula para $p_{s}$ :

$$
p_{s}(z)=\frac{1}{1+\exp \left[\alpha\left(\frac{\phi(z)}{\phi_{l}}-1\right)\right]}
$$

De la definición que dimos en términos de probabilidad, tenemos que la cota es la altitud $z$ para la que $p_{s}\left(z_{l}\right)=1 / 2$. Esta ecuación se cumple si $\phi\left(z_{l}\right)=\phi_{l}$, lo que nos permite interpretar el parámetro $\phi_{l}$ como el valor de la fase en la cota. El otro parámetro $\alpha$ determina la forma de la curva, así, p. ej., la pendiente en $\phi_{l}$ es más pronunciada cuanto mayor sea $\alpha$ (figura 6).

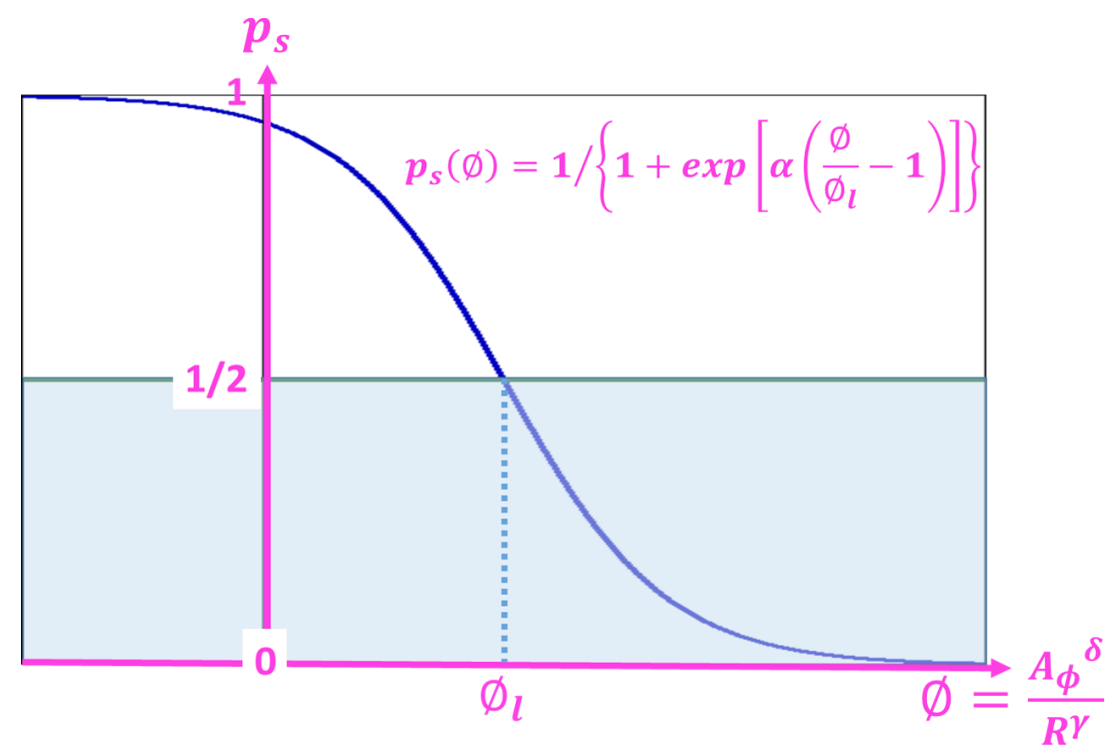

Figura 6. Probabilidad de nevada $p_{s}$ en función de la fase $\phi$. La probabilidad es del $50 \%$ en la cota de nieve $z_{l}: p_{s}\left(z_{l}\right)=1 / 2$.

$\underline{\text { Notas }}$

- La probabilidad de nevada en el suelo $(z=0)$ se obtiene directamente de [16]:

$$
\begin{gathered}
p_{s}(0)=\frac{1}{1+\exp \left[\alpha\left(\frac{\phi(0)}{\phi_{l}}-1\right)\right]} \\
\phi(0)=\left[\int_{0}^{z_{0}}\left(\frac{p}{p_{0}} \frac{T_{0}}{T}\right)^{b} t_{\phi}(t, h, p) d z\right]^{\delta} / R^{\gamma}
\end{gathered}
$$

- Como ya vimos en el cálculo de la proporción de copos, la probabilidad de nevada depende, a través de $\phi$, de la intensidad de precipitación. Hablaremos de probabilidad patrón y cota patrón cuando la intensidad sea de $1 \mathrm{~mm} / \mathrm{h}$. 


\section{APLICACIONES}

La teoría no ha sido verificada con datos experimentales por lo que los parámetros no están aún calibrados. Provisionalmente se considera $\phi_{1}=28$, valor que se obtiene, grosso modo, a partir de la regla práctica que considera que la cota viene a estar 200-300 m por debajo del inicio de la fusión y asociando esta regla a la intensidad patrón de $1 \mathrm{~mm} / \mathrm{h}$. Tras analizar también gráficas de probabilidad experimental de nevada, como ejemplo la de HäGGMARK, 1997, se adopta para $\alpha$ el valor provisional de $\alpha=\ln 20$.

Esta teoría se ha implementado en el ATAP tanto en los sondeos observados, como en los previstos de los modelos HRES-IFS del ECMWF y Harmonie-Arome, calculándose siempre con todos sus niveles (figura 7).

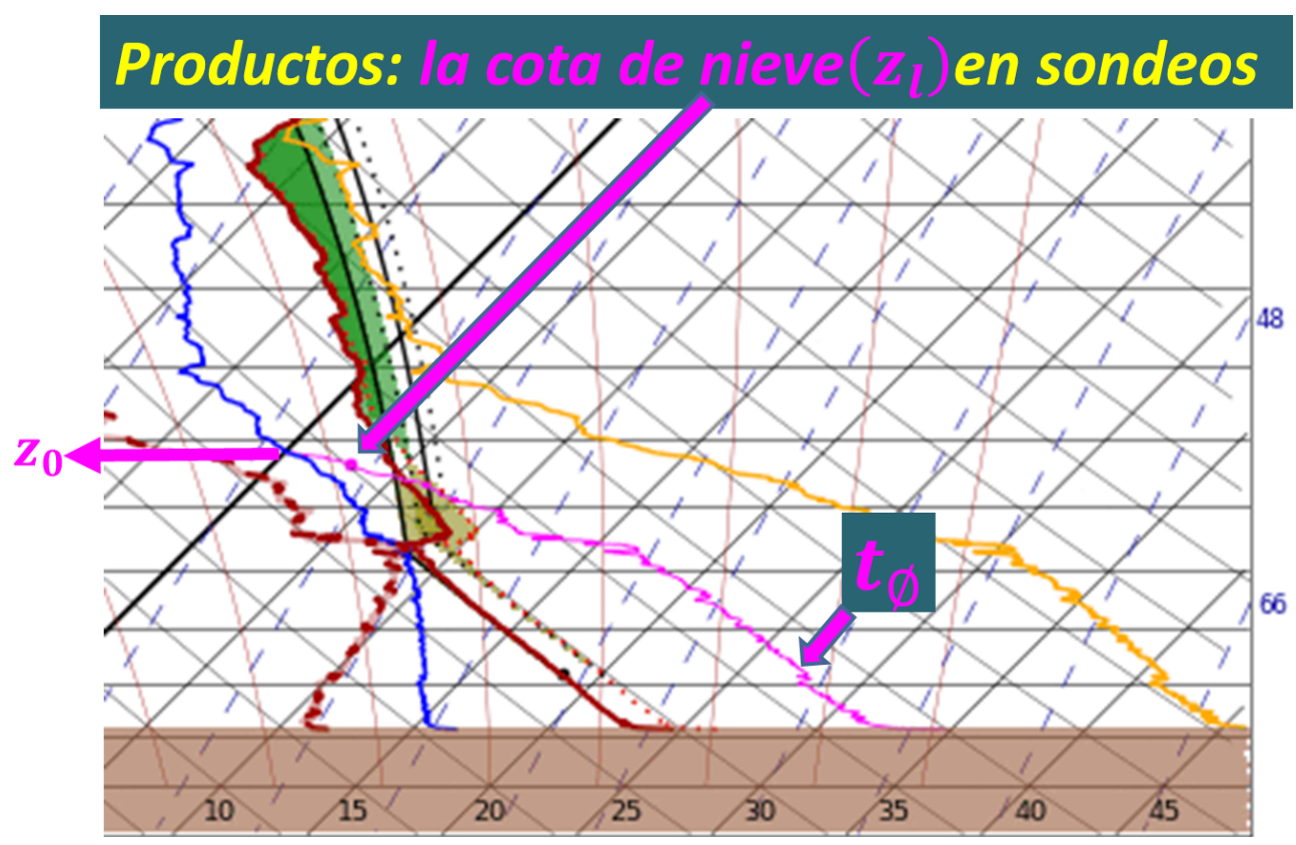

Figura 7. Temperatura de fase $t_{\phi}$ en un sondeo observado (línea magenta).

La bolita en la línea marca la cota de nieve $z_{l}$ para la intensidad de precipitación patrón de $1 \mathrm{~mm} / \mathrm{h}$.

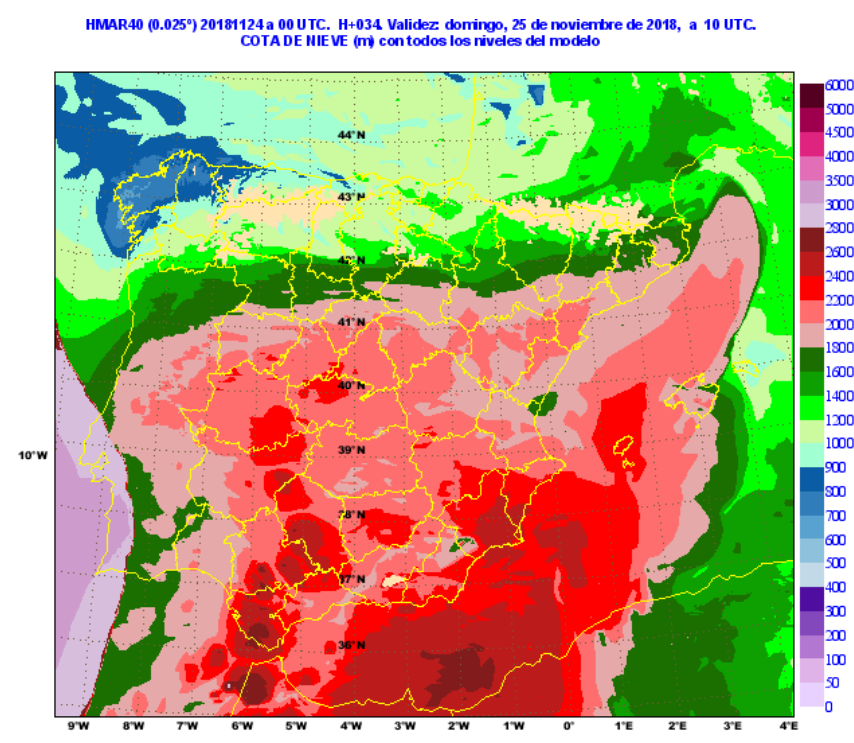

Figura 8. Cota de nieve patrón prevista por el modelo Harmonie-Arome calculada con todos sus niveles. Las zonas sin colorear indican posibilidad de nevada.
También se dibujan operativamente en el ATAP, en este caso a partir de sus niveles de presión, los mapas de la cota patrón de estos modelos, y próximamente, también con todos los niveles para HarmonieArome (figura 8).

La valoración subjetiva de los productos confirma la validez de la implementación con los parámetros provisionales. 


\section{CONCLUSIONES}

Se ha desarrollado un nuevo análisis de la transición de nieve a lluvia en el que se introducen las definiciones de temperatura y área de fase y, construida a partir de ellas, la de la fase $\phi$. El análisis se completa con una nueva formulación de la probabilidad de nevada y, asociado a esta, la definición probabilista de la cota de nieve.

La teoría está siendo ya aplicada a los sondeos observados y a los previstos por los modelos numéricos. También se aplica al dibujo de los mapas previstos de la cota de nieve patrón.

Como proyecto futuro se encuentra el de verificar y calibrar esta teoría con datos experimentales (sondeos, observaciones del tipo de precipitación y medidas de su intensidad), particularizándola también al caso de la nieve granulada. Otro proyecto próximo es el de aplicar la teoría a la estimación de la probabilidad de nevada a partir de datos observados en superficie.

\section{AGRADECIMIENTOS}

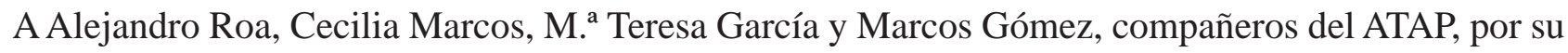
aportación en el desarrollo del proyecto y por el apoyo prestado en las charlas y discusiones relacionadas, que contribuyó a asentar las ideas expuestas en este artículo.

\section{REFERENCIAS}

ANDREAS, E. L., 2005. Handbook of Physical Constants and Functions for Use in Atmospheric Boundary Layer Studies. ERDC/CRREL Monogr. No. M-05-1, U. S. Army Cold Regions Research and Engineering Laboratory, 42 pp. Google Scholar.

Del Hoyo, J. et al., 2012. Comparación de los métodos de cota de nieve de AEMET.

HäGgmark, L., Ivarsson, K.-I. y Olofsson, P.-O. , 1997. MESAN Mesoskalig analys. SMHI. RMK Nr 75, mars 1997 (en sueco).

Hall, W. D. y PrupPACHER, H. R. 1976. The survival of ice particles falling from cirrus clouds in subsaturated air. Journal of the Atmospheric Sciences, 33, 1995-2006.

HARPOLD, A. A. et al., 2017. Rain or snow: hydrologic processes, observations, prediction, and research needs. Hydrol. Earth Syst. Sci., 21, 1-22.

Heymsfield, A. J., B Ansemer, A. y Twohy, C. H., 2007, Refinements to ice particle mass dimensional and terminal velocity relationships for ice clouds. Part I: Temperature dependence. J. Atmos. Sci., 64, 1047-1067.

Mitra, S. K., Vohl, O., Ahr, M. y Pruppacher, H. R., 1990. A wind tunnel and theoretical study of the melting behavior of atmospheric ice particles. IV: experiment and theory for snow flakes. Journal of the Atmospheric Sciences, 47 (5), 584-591.

Sekhon, R. S. y Srivastava, R. C., 1970. Snow spectra and radar reflectivity. J. Atmos. Sci., 27, 299-307.

Szyrmer, W. y Zawadzki, I., 1999. Modeling of the melting layer. Part I: Dynamics and microphysics. J. Atmos. Sci., 56, 3573-3592. 